
subsequent decision and action behaviors

* Equal contribution to the work

Running Head:

Decision and action influence subsequent decision and action behaviors

Corresponding author information:

\title{
David Thura
}

\author{
Lyon Neuroscience Research Center - Impact team \\ Inserm U1028 - CNRS UMR5292 - Lyon 1 University \\ 16 avenue du Doyen Jean Lépine, 69675 Bron, France \\ E-mail: david.thura@inserm.fr
}

\section{Manuscript information:}

Abstract: 253 words; Introduction, Methods and Discussion: 3094 words 


\section{Abstract}

34 In general, speed-accuracy tradeoff adjustments in decision-making have been studied separately

35 from those in motor control. In the wild however, animals coordinate their decision and action,

36 freely investing time in choosing versus moving given specific contexts. Recent behavioral studies

37 support this view, indicating that humans can trade decision time for movement time to maximize

38 their reward rate at the level of entire experimental sessions. Besides, it is established that choice

39 outcomes largely impact subsequent decisions. Crucially though, whether and how a decision also

40 influences the subsequent motor behavior, and whether and how a motor error influences the next

41 decision is currently unknown. Here we address these questions by analyzing trial-to-trial changes

42 of choice and motor behaviors in humans instructed to perform successive perceptual decisions

43 expressed with reaching movement whose duration was either bounded or unconstrained in

44 separate tasks. Results indicate that after a bad decision, subjects who were not constrained in their

45 action duration decided more slowly and more accurately. Interestingly, they also shortened their

46 subsequent movement duration by moving faster. Conversely, we found that movement errors not

47 only influenced the speed and the accuracy of the following movement, but those of the decision

48 as well. If the movement had to be slowed down, the decision that precedes that movement was

49 accelerated, and vice versa. Together, these results indicate that from one trial to the next, humans

50 are primarily concerned about determining a behavioral duration as a whole instead of optimizing

51 each of the decision and action speed-accuracy trade-offs independently of each other. 


\section{Introduction}

53 Choosing one action among several options and executing that action are usually considered as

54 two distinct functions, most often studied separately from each other (e.g. Franklin \& Wolpert,

55 2011; Ratcliff et al., 2016). However, recent behavioral studies indicate that decision and action

56 are instead tightly linked, sharing important principles and showing a high level of integration

57 during goal-oriented behavior (Choi et al., 2014; Cos et al., 2011; Haith et al., 2012; Morel et al.,

58 2017; Shadmehr et al., 2010, 2019; Shadmehr \& Ahmed, 2020; Yoon et al., 2018). For example,

59 human subjects decide faster and less accurately to focus on their actions when the motor context

60 in which a choice is made is demanding (Reynaud et al., 2020). Similarly, when the temporal cost

61 of a movement is significantly larger than usual, humans often reduce the duration of their

62 decisions to limit the impact of these time-consuming movements (Saleri Lunazzi et al., 2021).

63 Conversely, if the sensory evidence guiding the choice is weak and the deliberation takes time,

64 humans and monkeys shorten the duration of the movement expressing that choice (Thura, 2020;

65 Thura et al., 2014). Individuals thus seem to be primarily concerned about determining a global

66 behavior duration rather than optimizing decision and action durations separately, even if the

67 resulting decision or movement accuracy must slightly suffer. This "holistic-heuristic" policy may

68 serve what matters the most for decision-makers during successive decisions between actions, the

69 rate of reward (Balci et al., 2011; Carland et al., 2019; Thura, 2021).

70 Importantly, most of the adjustments mentioned above occur between blocks of tens to hundreds

71 of trials, depending on stable contexts favoring a fixed movement or decision speed-accuracy

72 trade-off. But can these adjustments also occur on shorter time scales, from trial to trial, depending

73 on the very local one's decision and motor performance? 
74 Indeed, performance history is known to exert a large influence on subsequent behavior (e.g.

75 Danielmeier \& Ullsperger, 2011; Jentzsch \& Dudschig, 2009; Urai et al., 2019). The most well-

76 known post-outcome adjustment is a reduction of behavior speed after committing an error,

77 namely post-error slowing (PES). PES is sometimes accompanied by changes in accuracy,

78 although conditions leading to PES-related increase or decrease of accuracy are still unclear

79 (Danielmeier \& Ullsperger, 2011; Fievez et al., 2021). Notably, post-outcome adjustments have

80 been mostly described as the effect of a choice on the decisional performance in the following trial

81 (Dutilh et al., 2012; Laming, 1979; Rabbitt \& Rodgers, 1977; Thura et al., 2017; Urai et al., 2019),

82 but the influence of a movement outcome on the motor performance in the following trial did not

83 receive the same attention (Ceccarini \& Castiello, 2018). Importantly, the consequences of either

84 a decision or a motor outcome on both subsequent decisions and movements have never been

85 investigated. These are important questions to address in order to further evaluate the level of

86 integration of the decision and the action functions during goal-directed behavior.

87 In the present report, we thus aim at investigating the consequences of a decision outcome on the next trial decision and motor performance. We also aim at analyzing the effect of a motor outcome

89 on the next trial decision and motor performance. Because we make the hypothesis that humans decide and act in a "holistic-heuristic" way, we predict that any adjustment due to a decision or a

91 motor outcome will be shared and integrated across the decision and the movement in the next

92 trial. This hypothesis also predicts that the integrated post-outcome adjustments will depend on

93 the capacity of the subject to "freely" share decision time for action time, and vice versa, if needed.

94 To test this hypothesis, we analyzed datasets from two recent studies of our group during which

95 human subjects made successive perceptual decisions between actions. In the first experiment

96 (Reynaud et al., 2020; Thura, 2020), participants could invest up to 3s in the decision process and 
97 had up to $800 \mathrm{~ms}$ to execute the reaching movement expressing a choice. In the other experiment

98 (Saleri Lunazzi et al., 2021), the decision component of the task was similar but subjects' reaching

99 duration was strictly bounded. By analyzing changes of several decision and motor parameters,

100 we found multiple context-dependent post-decision and post-movement outcome adjustments of

101 both subsequent decision and motor speed-accuracy tradeoffs.

102 Material and methods

103 Participants

104 Two groups of healthy, human subjects participated in the two experiments described in this report.

105 Twenty subjects (ages: 20-41; 16 females, 4 males; 18 right-handed) performed the free-movement

106 duration (FMD) task and thirty-one other subjects (ages: 18-36; 20 females, 11 males; 29 right-

107 handed) performed the constrained-movement duration (CMD) task. All gave their consent orally

108 before starting the experiment. The ethics committee of Inserm (IRB00003888) approved the

109 protocol on March $19^{\text {th }}$ 2019. Each participant performed two experimental sessions of the same

110 task. They received monetary compensation for completing each session (either $40 €$ for the FMD

111 task or $30 €$ for the CMD task).

112 Datasets

113 The decision and motor behaviors of these subjects have been described in three recent 114 publications reporting the effects of the decisional context on movement properties (Thura, 2020)

115 and the effects of the motor context on decision strategies (Reynaud et al., 2020; Saleri Lunazzi et 116 al., 2021). In these reports, subjects' behavioral adjustments are described either within a given

117 trial (i.e. the relation between a decision duration and the duration of the movement produced to 118 express that decision) or between specific conditions designed to set stable decision or motor 
A

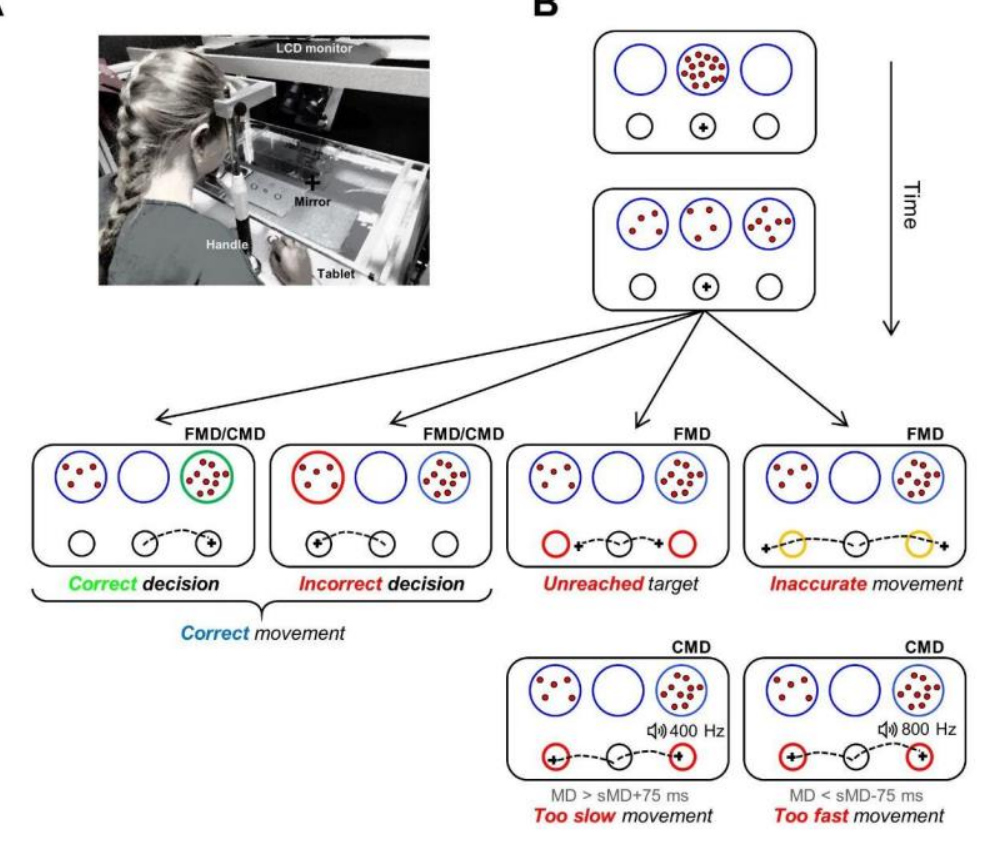

C

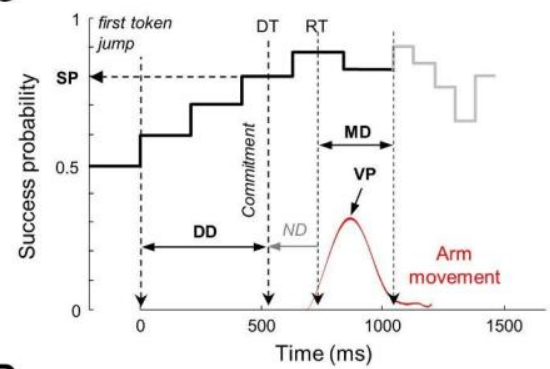

D

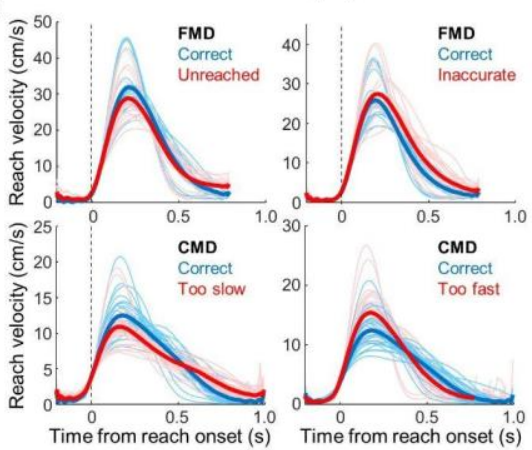

Figure 1. Methods. A. Experimental apparatus, common to both the FMD and CMD tasks. B. Time course of a trial in the decision task. Tokens jump one-by-one from the central decision circle to one of the two lateral ones. Subjects move a cursor from a central movement target to one of the two lateral ones to express their choice. All the decision and action outcomes are illustrated in bottom panels (please refer to the main text for details). MD: Movement duration; sMD: Spontaneous movement duration. C. Temporal profile of success probability (SP) in one example trial of the choice task. At the beginning of the trial, each target has the same success probability (0.5). When the first token jumps into one of the two potential targets (the most leftward vertical dotted line), the success probability of that target increases to $\sim 0.6$. Success probability then evolves with every jump. Subjects execute a reaching movement (red trace) to report their choice. Kinematic data allow to compute movement duration (MD) and movement peak velocity (VP). Non-decisional (ND) delays, determined in a separate reaction time task, allow to estimate decision duration (DD) and success probability (SP) at decision time. Only 10 out of 15 jumps are illustrated on this SP profile. D. Average reach velocity profiles aligned on reaching movement onset. Correct and "unreached" movements executed in the FMD task are compared in the top left panel; Correct and "inaccurate" movements executed in the FMD task are compared in the top right panel. Correct and "too slow" movements executed in the CMD task are compared in the bottom right panel. Correct and "too fast" movements executed in the CMD task are compared in the bottom left panel. 
143 The experimental apparatus (figure 1A), identical in the two tasks, as well as visual displays (figure

144 1B), are detailed and illustrated in the previous publications mentioned above (Reynaud et al., 145 2020; Saleri Lunazzi et al., 2021; Thura, 2020). The subjects sat in an armchair and made planar 146 reaching movements using a handle held in their dominant hand. A digitizing tablet (GTCO 147 CalComp) continuously recorded the handle horizontal and vertical positions (100 Hz with 0.013 $148 \mathrm{~cm}$ accuracy). Target stimuli and cursor feedback were projected by a LCD monitor onto a halfsilvered mirror suspended $26 \mathrm{~cm}$ above and parallel to the digitizer plane, creating the illusion that targets floated on the plane of the tablet. Participants were faced with a visual display consisting of three blue circles (the decision circles) placed horizontally at a distance of $6 \mathrm{~cm}$ from each other. In the central blue circle, 15 tokens were randomly arranged. Positioned below, three black circles, organized horizontally as well, defined the movement targets. The central black circle radius was $0.75 \mathrm{~cm}$. The size and location of the lateral black circles could vary in blocks of trials depending on the task. In the free-movement duration (FMD) task, that size was set to be either 0.75 or 1.5 $\mathrm{cm}$ of radius, and distance from the central circle was varied to be either 6 or $12 \mathrm{~cm}$ (as mentioned

157 above, effects of target size/position on subjects' behavior are not included in the present report).

158 In the constrained-movement duration (CMD) task, we analyzed trials for which the target size 159 was set to be $1.5 \mathrm{~cm}$ of radius and distance from the central circle was set to $6 \mathrm{~cm}$. (National Instruments), subjects initiated a trial by holding the handle into the black central circle

162 (starting position) for $500 \mathrm{~ms}$. Tokens then started to jump, one by one, every $200 \mathrm{~ms}$, in one of the two possible lateral blue circles. Subjects had to decide which of the two lateral blue circles

164 would receive the majority of the tokens at the end of the trial. They reported their decisions by 
moving the lever into the lateral movement target corresponding to the side of the chosen decision circle. Crucially, participants were allowed to make and report their choice at any time between the first and the last token jump. Once a target was reached, the remaining tokens jumped more quickly to their final circles (figure 1C, gray line), implicitly encouraging subjects to decide before all tokens had jumped to save time and increase their rate of reward at the session level. In the FMD task, tokens could speed up either a lot (a jump every $50 \mathrm{~ms}$ ) or a little (a jump every 150 ms) in given blocks of trials. These block-related effects are not included in the present report. In the CMD task, the remaining tokens jumped every $50 \mathrm{~ms}$.

In the free-movement duration (FMD) task, subjects had up to $800 \mathrm{~ms}$ to reach a target and report their choices. If no target was reached within $800 \mathrm{~ms}$, trials were classified as "unreached" trials, regardless of the direction of the movement with respect to the starting position. If the subject reached a target but failed to stop in it within $800 \mathrm{~ms}$, the trial was classified as "inaccurate" trial, regardless of the choice made, correct or incorrect (figure 1B).

In the constrained-movement duration (CMD) task, participants were instructed to reach a target within a 75-ms time interval around their spontaneous mean movement duration, computed in dedicated trials (please see Saleri Lunazzi et al., 2021 for details). So, if for a given subject we estimated a mean spontaneous reaching duration of $400 \mathrm{~ms}$ for the $6 \mathrm{~cm}$ long movements, then this subject had to report each of her/his choices by executing a movement whose duration was strictly bounded between 325 and 475 ms. In this CMD task, a trial was thus considered as a movement error trial when the subject did not meet these temporal constraints, even if the correct decision was made. We distinguished either "too slow" and "too fast" movement errors (figure 1B).

At the end of each trial of both tasks, a visual feedback about decision success or failure (the chosen decision circle turning either green or red, respectively) was provided to the subject after 
the last token jump, assuming a correct movement. In the FMD task, a movement error was indicated by visual feedback. The chosen movement target turned orange in "inaccurate" trials, the two movement targets turned red in "unreached" trials. In the CMD task, a movement error was indicated by both a visual and a 500 ms audio feedback (both movement targets turned red and an 800 or $400 \mathrm{~Hz}$ sound indicating that movement was too fast or too slow, respectively, was played). Subjects had to make a specific number of correct trials (either 320 trials in the FMD task or 160 trials in the CMD task), indirectly motivating them to optimize successes per unit of time.

Finally, subjects also performed in each of the two sessions of both tasks a simple delayed-reaching task (DR task, 100 trials for subjects performing the FMD task and 20 trials for subjects who performed the CMD task). This DR task was identical to the choice task described above, except that there was only one lateral decision circle displayed at the beginning of the trial (either at the right or at the left side of the central circle with $50 \%$ probability). All tokens moved from the central circle to this unique circle at a GO signal occurring after a variable delay (1000 $\pm 150 \mathrm{~ms})$. This DR task was used to estimate the sum of the delays attributable to response initiation (i.e. non-decision delays).

\section{Subsets of trials based on decision and movement outcomes}

We first defined three subsets of trials common to both tasks (FMD and CMD), based on decision or movement outcomes: (1) "Correct decision" trials, when the subject chose the correct target and reported her/his choice with a correct movement; (2) "Incorrect decision" trials, if the participant chose the incorrect target with a correct movement. Note that for these two subsets, bad movement trials are excluded because no feedback was provided to the subject to indicate whether or not she/he chose the correct target. By contrast, a salient feedback was provided at the end of the trial 
to indicate the movement error (see above and figure 1B); (3) "Correct movement" trials, when

211 the subject adequately reached the correct or the incorrect target.

212 We defined two other subsets of trials based on movement errors in the FMD task specifically: (1)

213 "Unreached" trials, when the subjects failed to reach a target (correct or incorrect) before the end

214 of the movement duration deadline $(800 \mathrm{~ms})$; (2) "Inaccurate" trials, when the subjects reached a

215 target (correct or incorrect) but failed to stop in it.

216 Finally, two subsets of trials were defined based on movement errors in the CMD task specifically:

217 (1) "Too fast movement" trials and (2) "Too slow movement" trials, when the subjects reached a

218 target (correct or incorrect) before the minimum instructed duration time and after the maximum

219 instructed duration time, respectively.

220 Data analysis

221 Data were analyzed off-line using custom-written MATLAB (MathWorks) and R (https://www.r-

222 project.org/) scripts. Reaching horizontal and vertical positions were first filtered using polynomial

223 filters and then differentiated to obtain a velocity profile. Onset and offset of movements were then

224 determined using a $3.75 \mathrm{~cm} / \mathrm{s}$ velocity threshold. Reaching movement duration (MD), peak

225 velocity (VP) and amplitude (Amp) were respectively defined as the duration, the maximum

226 velocity value and the Euclidean distance between these two events (figure 1C). Reaching

227 movement accuracy was defined as the Euclidian distance separating the target center from the

228 movement endpoint location (CED).

229 Decision duration (DD) was computed as the duration between the first token jump and the time

230 at which subjects committed to their choice (figure 1C). To estimate this commitment time in each

231 trial, we detected the time of movement onset as mentioned above, defining the subject's reaction 
232 time, and subtracted from it her/his mean sensory-motor delays estimated based on her/his reaction

233 times in the DR task performed the same day and in the same condition.

234 To assess the influence of sensory evidence on subjects' choices, we computed the success

235 probability profile of each trial experienced by participants with respect to the chosen target, as

236 well as their decision success probability (SP) at the time of commitment time (figure 1C), using

237 Equation 1. For instance, for a total of 15 tokens, if at a particular moment in time the target chosen

238 by the subject contains $\mathrm{N}_{\text {chosen }}$ tokens, whereas the other target contains $\mathrm{N}_{\text {other }}$ tokens, and there are

$239 \mathrm{~N}_{C}$ tokens remaining in the center, then the probability that the chosen target will ultimately be the

240 correct one, i.e. the subject's success probability (SP) at a particular time is as follows:

$$
p\left(\text { Chosen } \mid N_{\text {chosen }}, N_{\text {other }}, N_{C}\right)=\frac{N_{C} !}{2^{N_{C}}} \sum_{k=0}^{\min \left(N_{C}, 7-N_{\text {other }}\right)} \frac{1}{k !\left(N_{C}-k\right) !}
$$

241 To ensure that the difficulty of decisions was homogeneous among subjects and experimental

242 conditions, we controlled the sequence of trials experienced by each participant in each session of

243 both tasks. Especially, we interspersed among fully random trials ( $20 \%$ of the trials in which each

244 token is $50 \%$ likely to jump into the right or the left lateral circle) three special types of trials, easy,

245 ambiguous and misleading, characterized by particular temporal profiles of success probability.

246 Subjects were not told about the existence of these trials. Please refer to Reynaud et al., 2020 and

247 Saleri Lunazzi et al., 2021 for a detailed description of these trial types and their proportions in the

$248 \quad$ FMD and CMD tasks.

249 To assess the impact of the outcome of each trial $i$ on the decision and motor behavior of trial $i+1$,

250 we calculated the difference of movement velocity peak $(\Delta \mathrm{VP})$, duration $(\Delta \mathrm{MD})$, amplitude

$251(\triangle \mathrm{Amp})$, accuracy $(\triangle \mathrm{CED})$, and the difference of decision duration $(\Delta \mathrm{DD})$ and success probability 
252 ( $\Delta \mathrm{SP}$ ) between them (e.g. $\left.\Delta V P=V P_{i+1}-V P_{i}\right)$. We then calculated for each subject the average

253 of each variable with respect to trial $i$ outcome.

254 Statistics

255 To determine whether the behavioral adjustment from one trial to the following $(\Delta \mathrm{VP}, \Delta \mathrm{MD}$, $256 \Delta \mathrm{Amp}, \triangle \mathrm{CED}, \Delta \mathrm{DD}$ and $\Delta \mathrm{SP})$ differs significantly from 0 in the different outcome conditions at

257 the population level, we used one-sample Wilcoxon signed rank tests. The Levene's test is used to 258 test if the distributions of the post-correct and post-error decision and motor variables have equal 259 variances. To directly investigate the relationship between motor $(\Delta \mathrm{VP}, \Delta \mathrm{MD}, \Delta \mathrm{Amp}, \Delta \mathrm{CED})$ and 260 decision $(\triangle \mathrm{DD}, \Delta \mathrm{SP})$ adjustments following different outcomes, Pearson's correlation tests were 261 used. For all statistical tests, the significance level is set to 0.05. Unless stated otherwise, data are 262 reported as medians across the population. To estimate the difference between the average success 263 probability profiles of two trial subsets (e.g. correct decision trials versus post-correct decision 264 trials), we computed the distance between the two profiles ( 1 and 2$)$ from token jump ( $j) \# 1$ to \#15 265 as the following chi-squared metric:

$$
\chi^{2}=\sum_{j=1}^{15} \frac{\left(y_{1, j}-y_{2, j}\right)^{2}}{\sigma_{j}^{2}}
$$

266 where $y_{1}$ and $y_{2}$ are the two SP profiles, averaged across subjects, and $\sigma_{j}^{2}$ is the mean squared

267 variance of the SP profiles, such as $\sigma_{j}^{2}=\frac{1}{2}\left(\sigma_{1, j}^{2}+\sigma_{2, j}^{2}\right)$. 
270 We first describe the impact of the decision outcome (correct or incorrect choice) on participants'

271 subsequent decisional behavior when the motor temporal constraints were low (FMD task). As

272 shown in figure 2A, subjects' decision duration was significantly increased compared to a previous

273 incorrect decision (median $\Delta \mathrm{DD}=+70.6 \mathrm{~ms}$, Wilcoxon signed rank test, $\mathrm{Z}=2.4, \mathrm{p}=0.015$ ). This

274 slowdown of decision-making was observed despite that trials following an incorrect choice were

275 easier, as can be seen on the averaged success probability (SP) profiles of the two trial subsets

$276 \quad\left(\chi^{2}=2119\right.$, inset in figure 2A, top right panel; Suppl. figure 1 illustrates the SP profiles of the same

277 trials computed with respect to the correct target). As a consequence, subjects' SPs at decision

278 time were increased following incorrect decisions $(\Delta \mathrm{SP}=+0.09, \mathrm{Z}=3.9, \mathrm{p}<0.001)$. By contrast, no

279 significant difference of decision duration $(\Delta \mathrm{DD}=-2.8 \mathrm{~ms})$ was observed following a correct

280 decision. Together, this first analysis demonstrates that most subjects used a post-error slowing

281 strategy to decide in this task, as can be seen when decision durations following either a correct or

282 a bad choice are directly compared (suppl. figure 2). Interestingly, subjects did not adjust their

283 decision duration following a correct trial despite that these trials were on average slightly more

284 difficult $\left(\chi^{2}=207\right.$, inset in figure 2 A, bottom right panel). Participants' success probability thus

285 slightly decreased after a correct choice $(\Delta \mathrm{SP}=-0.02, \mathrm{Z}=-3.9, \mathrm{p}<0.001)$, indicating that they

286 committed to a decision with less sensory evidence after a correct trial. 
A
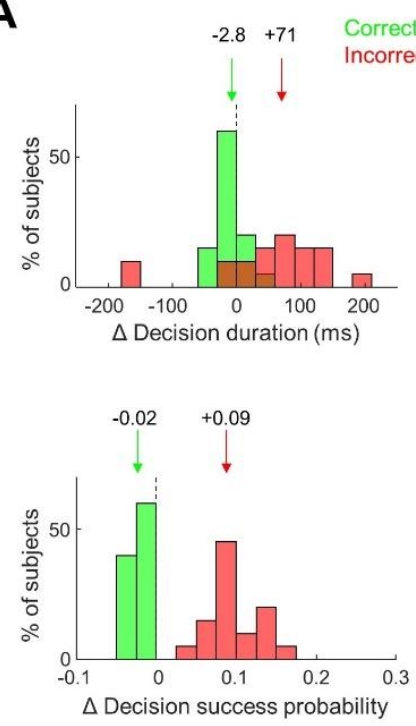

Correct decision

ct decision
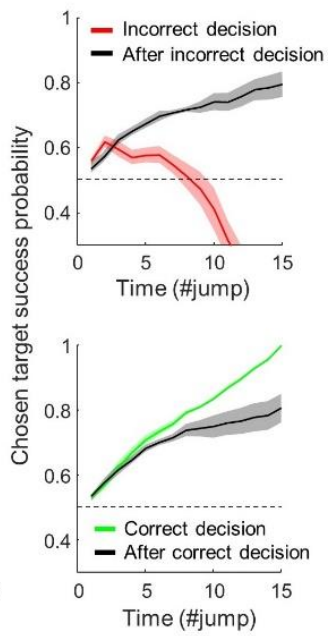

B
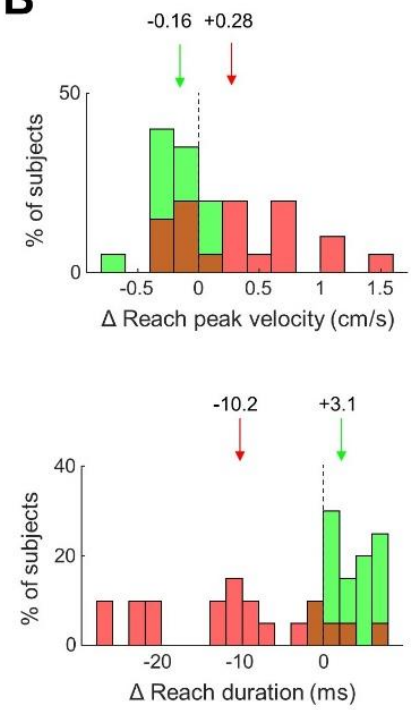

301

Figure 2. Effect of a decision outcome on the subsequent decision and motor behaviors in the FMD task. A. Left panels: Distribution and comparison of decision duration (top) and success probability (bottom) adjustments depending on the decision outcome of the previous trial (after a correct trial in green and after an incorrect decision in red). Arrows mark the population medians whose values are reported above. The dotted black line indicates zero difference between the trial $i$ and $i+1$. If $\Delta$ is positive, there is a post-outcome increase for a given metric $\mathrm{X}\left(\mathrm{X}_{i+1}-\mathrm{X}_{i}>0\right)$ whereas a negative $\Delta$ value indicates a decrease of that metric. Right panel, top: Comparison of the average \pm SD success probability profiles between trials whose decision was incorrect (red solid line) and trials following an incorrect choice (black dotted line), computed across subjects with respect to the target they chose. Right panel, bottom: same comparison between correct decision trials (green solid line) and trials following a correct decision (black solid line). B: Same analysis as in A, left panels, for the post-decision outcome adjustments computed for movement peak velocity (top) and duration (bottom).

We next investigate whether or not decision outcomes impact motor behavior as well. We found that following incorrect decisions, subjects made overall faster movements $(\Delta \mathrm{VP}=+0.28 \mathrm{~cm} / \mathrm{s}$, $\mathrm{Z}=2.4, \mathrm{p}=0.01)$, thus reducing their reaching duration $(\Delta \mathrm{MD}=-10.2 \mathrm{~ms}, \mathrm{Z}=3.5, \mathrm{p}<0.001$, figure $2 \mathrm{~B})$ despite a decrease of amplitude $(\Delta \mathrm{Amp}=-0.08 \mathrm{~cm}, \mathrm{Z}=-3, \mathrm{p}=0.002$, suppl. figure 3$)$. We also observed at the population level an increase of movement inaccuracy after an incorrect choice $(\Delta \mathrm{CED}=+0.06 \mathrm{~cm}, \mathrm{Z}=3.1, \mathrm{p}=0.002)$, but this effect was also observed for trials following correct decisions $(\triangle \mathrm{CED}=+0.06 \mathrm{~cm}, \mathrm{Z}=3.9, \mathrm{p}<0.001$, suppl. figure 3). 
In the free-movement duration (FMD) task, we distinguished two types of movement error: "Inaccurate" trials, when a target was reached but subjects failed to stop in it, and "unreached" trials, when the subject failed to reach a target before the movement duration deadline ( $800 \mathrm{~ms}$ in the FMD task). "Inaccurate" movements were thus on average faster $(26.8$ vs $26 \mathrm{~cm} / \mathrm{s})$, larger in amplitude (9.2 vs $8.7 \mathrm{~cm}$ ) and longer (557 vs $530 \mathrm{~ms}$ ) compared to correct movements (figure 1D, an inaccurate movement (suppl. figure 4A). probabilities are much variable and distributed across the population in trials following an

322 inaccurate movement compared to trials following a correct movement $(\mathrm{SD}=182$ versus $4.6 \mathrm{~ms}$, 323 respectively; Levene's test, $\mathrm{F}=21.7, \mathrm{p}<0.0001)$. In terms of medians, decision durations following 324 an inaccurate movement were overall shorter compared to trials for which a movement was 325 inaccurate, but this difference is not significant $(\triangle D D=-39 \mathrm{~ms})$. We also observed a slight but 326 significant increase of decision success probability $(\Delta \mathrm{SP}=+0.04, \mathrm{Z}=2.8, \mathrm{p}=0.005)$ following 327 inaccurate movements, possibly because of the slightly higher SP profile of trials following 328 inaccurate movements compared to the error movement trials $\left(\chi^{2}=21.8\right.$, figure $3 \mathrm{~A}$, bottom right 329 panel). To directly assess the relationship between motor and decision adjustments due to inaccurate movements, we computed linear regressions between all differences of motor $(\Delta \mathrm{VP}$, $\triangle \mathrm{MD}, \Delta \mathrm{CED}, \Delta \mathrm{Amp})$ and decision $(\Delta \mathrm{DD}, \Delta \mathrm{SP})$ metrics. We found a significant negative

332 correlation between $\triangle \mathrm{MD}$ and $\triangle \mathrm{DD}$ (Pearson correlation, $\mathrm{R}=-0.62, \mathrm{p}=0.003$ ) and a significant 

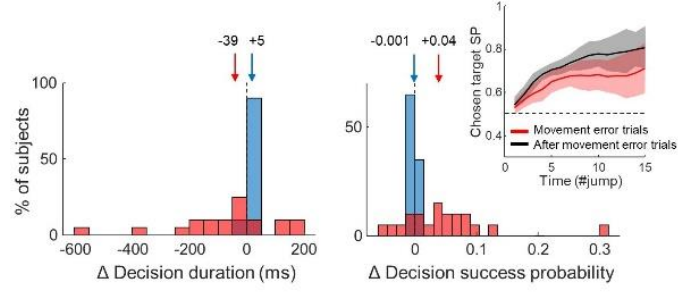

B
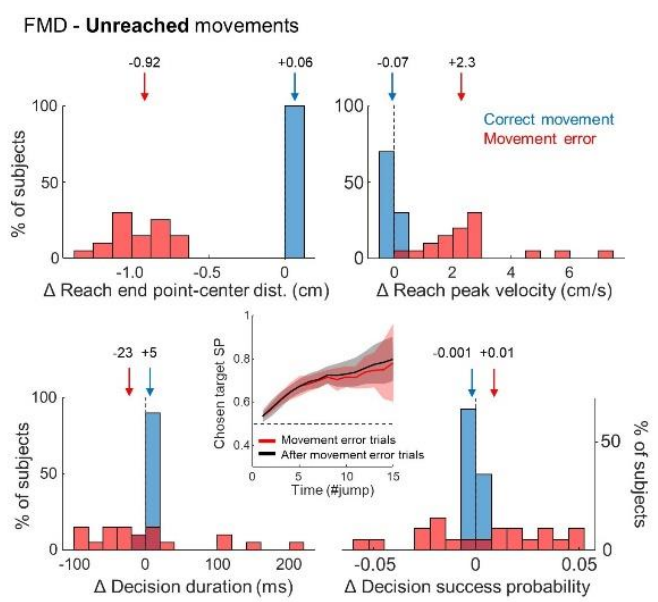

Figure 3. Effect of movement errors on subsequent motor and decision behaviors in the FMD task. A. Top: Distribution and comparison of reaching movement end-point center distance (left) and peak velocity (right) adjustments depending on the movement outcome in the previous trial (after a correct movement in blue and after an "inaccurate" movement in red). Bottom: Distribution and comparison of decision duration (left) and success probability (right) adjustments depending on the movement outcome in the previous trial (after a correct movement in blue and after an "inaccurate" movement in red). The inset illustrates the average \pm SD success probability profiles of inaccurate movement trials (red) and postinaccurate movement trials (black), computed across subjects. B. Same as A for the "unreached" trials.

While various reasons could lead to "unreached" trials, we noticed that overall, movements in these trials were on average slower compared to correct movements (figure 1D, top left panel). Following these unreached trials, subjects significantly increased their movement accuracy in the next trial $(\triangle \mathrm{CED}=-0.92 \mathrm{~cm}, \mathrm{Z}=-3.9, \mathrm{p}<0.001)$ by increasing their reaching velocity peak $(\Delta \mathrm{VP}=+2.3 \mathrm{~cm} / \mathrm{s}, \mathrm{Z}=3.9, \mathrm{p}<0.001$, figure $3 \mathrm{~B}$, top panels). They also increased their reaching amplitude $(\Delta \mathrm{Amp}=+1 \mathrm{~cm}, \mathrm{Z}=3.9, \mathrm{p}<0.001)$ and duration $(\Delta \mathrm{MD}=+20 \mathrm{~ms}, \mathrm{Z}=2.9, \mathrm{p}=0.004)$ compared to the previous erroneous trials (suppl. figure 4C). 
After an "unreached" movement, we did not observe significant adjustments of the decisional behavior in the next trial at the population level $(\Delta \mathrm{DD}=-23 \mathrm{~ms}, \Delta \mathrm{SP}=+0.01)$, although distributions of mean decision durations and success probabilities are broader in trials following an unreached movement compared to trials following a correct movement ( $\mathrm{SD}=83$ versus $4.6 \mathrm{~ms}$; Levene's test, $\mathrm{F}=25, \mathrm{p}<0.0001$ figure $3 \mathrm{~B}$, bottom panels). We found however a significant positive correlation between the adjustment of peak velocities $(\Delta \mathrm{VP})$ following an unreached movement trial and the adjustment of decision durations $(\triangle \mathrm{DD})$ in the same condition $(\mathrm{R}=0.48, \mathrm{p}=0.04$, suppl. figure $4 \mathrm{D})$, indicating that participants who increased their movement speed the most after an unreached movement trial also increased their decision duration the most.

The previous paragraphs describe behavioral adjustments of subjects performing the freemovement duration (FMD) task. In the following lines, we report the same analyses applied on a dataset collected in the constrained movement duration (CMD) task. In this task, the duration of a movement executed to report a choice was strictly bounded (see methods), increasing the difficulty of the motor aspect of the task. In the FMD task, the average error percentage was $25 \%$, among which $18 \%$ of decisional errors and $7 \%$ of movement errors. In the CMD task however, the average error percentage was $50 \%$, with only $12 \%$ of decisional errors but $38 \%$ of movement errors.

371 We first assessed whether a decision outcome influenced the subsequent decision behavior in the

372 CMD task. As shown in figure 4A, the slowdown of decisions observed following incorrect 373 choices in the FMD task was not found in the CMD task ( $\Delta \mathrm{DD}=-5 \mathrm{~ms})$. Subsequent decision 374 success probabilities were increased following incorrect decisions $(\Delta \mathrm{SP}=+0.12, \mathrm{Z}=4.8, \mathrm{p}<0.001)$, 375 an adjustment likely due to post-incorrect decision trials that were easier compared to incorrect 376 decision trials $\left(\chi^{2}=767\right.$, inset in figure 4 A, right panel). Despite that decision outcomes did not 
377 influence the next decision in the CMD task, we observed that following incorrect choices,

378 participants increased their reaching velocity peak $(\Delta \mathrm{VP}=+0.24 \mathrm{~cm} / \mathrm{s}, \mathrm{Z}=3, \mathrm{p}=0.003)$, leading to a

379 decrease of movement duration $(\Delta M D=-13 \mathrm{~ms}, \mathrm{Z}=-3.4, \mathrm{p}<0.001$, figure $4 \mathrm{~B})$.
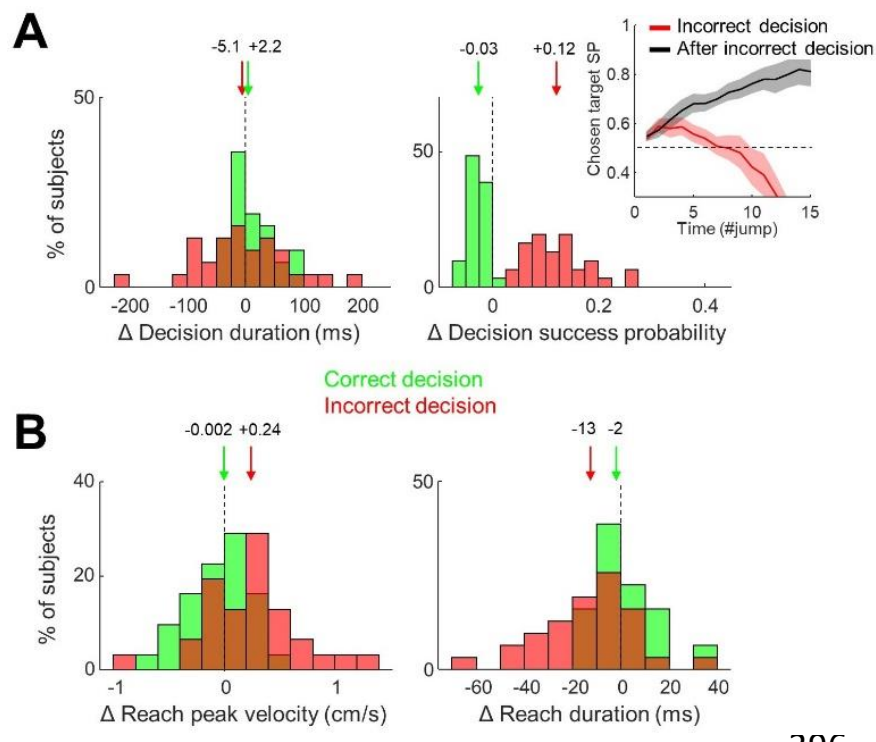

Correct decision

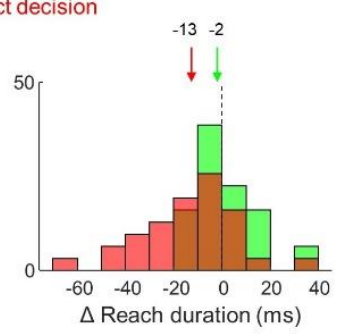

Figure 4. Effect of decision outcomes on subsequent decision and motor behaviors in the CMD task. A. Distribution and comparison of decision duration (left) and success probability (right) adjustments depending on the decision outcome in the previous trial (after a correct decision in green and after an error decision in red). The inset illustrates the average \pm SD success probability profiles of incorrect decision trials (red) and post-incorrect decision trials (black), computed across subjects. B: Distribution and comparison of reaching peak velocity (left) and duration (right) adjustments depending on the decision outcome in the previous trial (same conventions as in A).

In the last two paragraphs, we investigate the consequences of a movement error outcome in the CMD task, i.e. too fast or too slow movements, on subjects' behavior in the next trial. As expected, following too fast movements, participants significantly decreased their reaching velocity peak $(\Delta \mathrm{VP}=-3.1 \mathrm{~cm} / \mathrm{s}, \mathrm{Z}=-4.8, \mathrm{p}<0.001)$, leading to an increase of movement duration $(\Delta \mathrm{MD}=+62 \mathrm{~ms}$, $\mathrm{Z}=4.8, \mathrm{p}<0.001$, figure $5 \mathrm{~A}$, top panels). This adjustment was accompanied by a decrease of amplitude $(\triangle \mathrm{Amp}=-0.35 \mathrm{~cm}, \mathrm{Z}=-4.8, \mathrm{p}<0.001)$ and a decrease of accuracy $(\triangle \mathrm{CED}=+0.2 \mathrm{~cm}$, $\mathrm{Z}=4.8$, $\mathrm{p}<0.001$, suppl. figure $5 \mathrm{~A}$ ). The duration of decisions at the population level was significantly decreased following too fast movements in the CMD task $(\triangle \mathrm{DD}=-79 \mathrm{~ms}, \mathrm{Z}=-3.7$, $\mathrm{p}<0.001$, figure 5A, bottom left panel). Crucially, this adjustment is not due to a difference of decision difficulty between the two trial subsets $\left(\chi^{2}=1.2\right.$, inset in figure $5 \mathrm{~A}$, bottom right panel), 
trials.

A
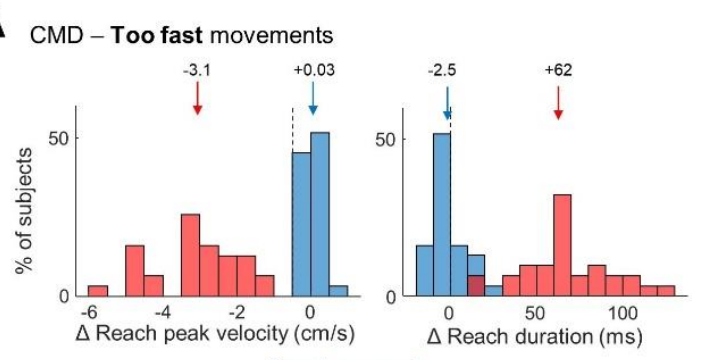

Correct movement

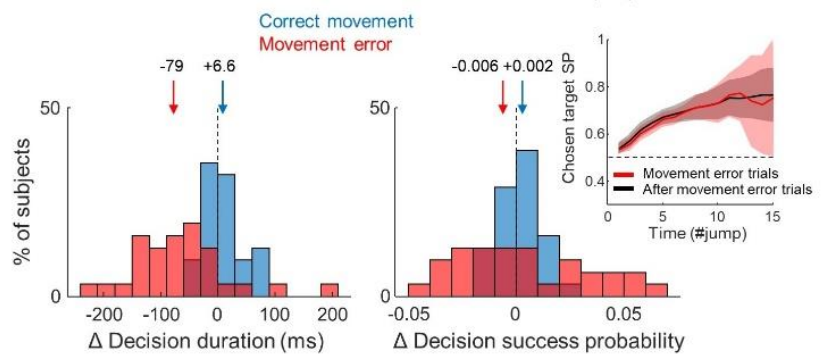

B
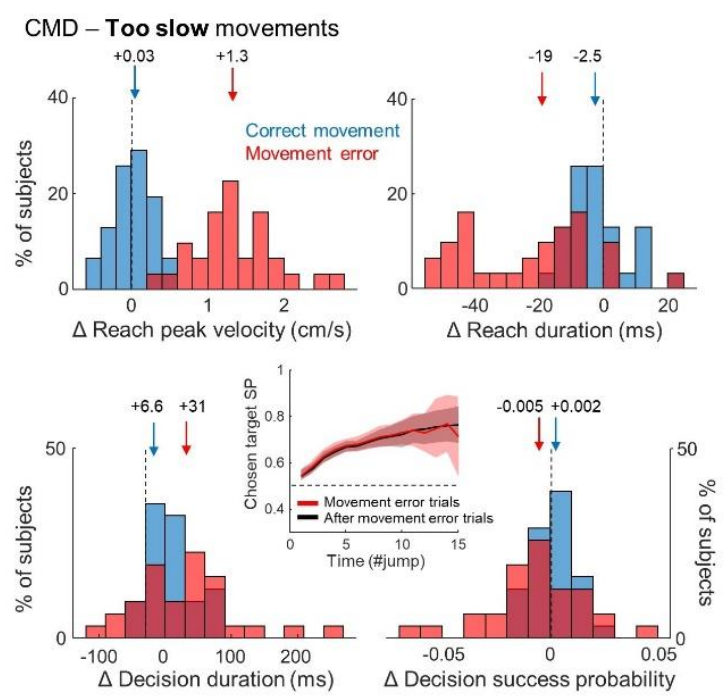

410

411

412

413

414

415

416

417

418

419

420

421

422

423

424

425

426

Figure 5. Effect of movement errors on subsequent motor and decision behaviors in the CMD task. A. Top: Distribution and comparison of reaching movement peak velocity (left) and duration (right) adjustments depending on the movement outcome in the previous trial (after a correct movement in blue and after a too fast movement in red). Bottom: Distribution and comparison of decision duration (left) and success probability (right) adjustments depending on the movement outcome in the previous trial (same convention as above). The inset illustrates the average \pm SD success probability profiles of too fast movement trials (red) and post-too fast movement trials (black), computed across subjects. B. Same as A for too slow movement trials in the CMD task.

After too slow movements, participants unsurprisingly increased their movement velocity peak $(\Delta \mathrm{VP}=+1.3 \mathrm{~cm} / \mathrm{s}, \mathrm{Z}=4.8, \mathrm{p}<0.001)$, which reduced reaching durations $(\Delta \mathrm{MD}=-19 \mathrm{~ms}, \mathrm{Z}=-4.4$, $\mathrm{p}<0.001$, figure $5 \mathrm{~B}$, top panels). Movement amplitude $(\Delta \mathrm{Amp}=+0.3 \mathrm{~cm}, \mathrm{Z}=4.8, \mathrm{p}<0.001)$ and accuracy $(\triangle \mathrm{CED}=-0.29 \mathrm{~cm}, \mathrm{Z}=-4.8, \mathrm{p}<0.001)$ were also significantly increased (suppl. figure $5 \mathrm{~B})$. Notably, following too slow movements, the duration of decisions was in this case significantly increased $(\triangle \mathrm{DD}=+31 \mathrm{~ms}, \mathrm{Z}=2.1, \mathrm{p}=0.038)$, despite no difference between the SP profiles in the tow trial subsets $\left(\chi^{2}=0.5\right)$, without a significant modulation of decision SP (figure $5 \mathrm{~B}$, bottom panels). 
427

428

429

430

431

432

433

434

435

436

437

438

439

440

441

442

443

444

447 CMD task concerned mostly movements, and decision error rates were similar in the two tasks.

448 We thus believe that the lack of decision-related PES in the CMD task mainly relates to the strict

449 duration constraints imposed on movements in this task (see below). 
We also observed the expected, yet very robust, post-movement error adjustments in participants' motor behavior. Generally, effects of behavior history on subsequent behavior have been investigated by means of cognitive tasks (Dutilh et al., 2012; Notebaert et al., 2009; Rabbitt \&

453 Rodgers, 1977), limiting the analysis to pre-movement processes (but see Ceccarini \& Castiello, 454 2018). The present report describes, to our knowledge, the first analysis addressing the impact of 455 decision and action outcomes on both the decision and action executed in the following trial. This 456 is important because in most everyday life choices, decisions and movements expressing these 457 choices are temporally linked, constituting a continuum separating an event from a potential 458 reward (Cisek, 2007).

459 We found that after a slower choice made in response to a decision error, movement duration, if 460 unconstrained, is reduced. This result is consistent with recent reports in both human and non461 human primates showing that within blocks of trials defined by specific speed-accuracy tradeoff 462 (SAT) properties, long decisions are expressed with vigorous, short movements (Thura, 2020; 463 Thura et al., 2014). We show here that this policy can be established on a shorter time scale, from 464 trial to trial, based on subject's previous trial outcomes.

465 Conversely, we found that when participants had to correct a bad movement, they not only 466 adequately adjusted their movements in the following trial, but they also altered the decision made 467 in this following trial, prior to the corrected movement expressing that choice. This observation is 468 at first sight consistent with several studies showing that the cost of a movement executed to report 469 a choice influences that choice in a given trial (Burk et al., 2014; Hagura et al., 2017; Marcos et 470 al., 2015). But it actually differs by demonstrating for the first time the capacity of humans to 471 preemptively compensate for a movement correction due to a motor error by altering the 472 deliberation process of the post-error trial, before the execution of the corrected movement. 
473 A possible functional interpretation of the reduction of movement duration accompanying a

474 decision-related PES (in the FMD task) is that subjects aimed at compensating the extra time

475 devoted to deliberation by executing faster movements, even if shortening movement duration

476 usually leads to a slight decrease of accuracy. In ecological scenarios, individuals are often free to

477 adjust the time they invest in deciding versus moving, and movements are parametrized following

478 "economic" rules (e.g. Shadmehr et al., 2019), allowing to optimize what matters the most for

479 individuals during successive choices, the rate of reward (Balci et al., 2011; Bogacz et al., 2010;

480 Carland et al., 2019).

481 In agreement with a reward rate maximization account, when a movement was corrected by

482 increasing or decreasing its duration, most participants decreased or increased their decision

483 duration, respectively, within the same trial. This is consistent with our previous reports in which

484 compensatory effects are described across blocks of tens of trials defined by specific motor SAT

485 constraints (Reynaud et al., 2020; Saleri Lunazzi et al., 2021). This suggests that one can flexibly

486 share temporal resources between the decision and the action processes depending on both global

487 and local contexts, even if these processes must slightly suffer in terms of accuracy (i.e. a good

488 enough, or heuristic, approach, Gigerenzer \& Gaissmaier, 2011). According to this mechanism,

489 the absence of decision-related PES when movement duration was strictly bounded would mean

490 that subjects anticipated that they could not compensate for a potential extension of their decision

491 duration following a bad choice during the movement phase, discouraging them to slow down their

492 decisions after a decision error. Intriguingly, they still produced faster and shorter movements after

493 a bad choice, indicating here an adjustment of movement duration that does not depend on the

494 decision determining this movement. It is possible that in this specific task where errors were 
495 frequent $(\sim 50 \%)$, subjects aimed at limiting the waste of time due an erroneous trial by moving

496 slightly faster in the next trial despite the strict constraints imposed on movement duration.

497 Taken together, the present results indicate that following both decision and movement errors,

498 humans are primarily concerned about determining a behavioral duration as a whole instead of 499 optimizing each of the decision and action speed-accuracy trade-offs independently of each other, 500 probably with the goal of maximizing their success rate. was preregistered. Perception, \& Psychophysics, 73(2), 640-657. https://doi.org/10.3758/s13414-010-0049-

Bandura, A., \& Locke, E. A. (2003). Negative self-efficacy and goal effects revisited. Journal of Applied Psychology, 88(1), 87-99. https://doi.org/10.1037/0021-9010.88.1.87

Bogacz, R., Hu, P. T., Holmes, P. J., \& Cohen, J. D. (2010). Do humans produce the speedaccuracy trade-off that maximizes reward rate? Quarterly Journal of Experimental

Burk, D., Ingram, J. N., Franklin, D. W., Shadlen, M. N., \& Wolpert, D. M. (2014). Motor Effort https://doi.org/10.1371/journal.pone.0092681 
517 Carland, M. A., Thura, D., \& Cisek, P. (2019). The Urge to Decide and Act: Implications for Brain Function and Dysfunction. The Neuroscientist, 107385841984155. https://doi.org/10.1177/1073858419841553

520

521

522

523

524

525

526

527

528

529

530

531

532

533

534

535

536

537

538

13. https://doi.org/10.1016/j.cognition.2018.05.026

Choi, J. E. S., Vaswani, P. A., \& Shadmehr, R. (2014). Vigor of Movements and the Cost of Time in Decision Making. Journal of Neuroscience, 34(4), 1212-1223. https://doi.org/10.1523/JNEUROSCI.2798-13.2014

Cisek, P. (2007). Cortical mechanisms of action selection: The affordance competition hypothesis. Philosophical Transactions of the Royal Society B: Biological Sciences, 362(1485), 1585-1599. https://doi.org/10.1098/rstb.2007.2054

Cos, I., Bélanger, N., \& Cisek, P. (2011). The influence of predicted arm biomechanics on decision making. Journal of Neurophysiology, 105(6), 3022-3033. https://doi.org/10.1152/jn.00975.2010

Danielmeier, C., \& Ullsperger, M. (2011). Post-Error Adjustments. Frontiers in Psychology, 2, 233. https://doi.org/10.3389/fpsyg.2011.00233

Dutilh, G., Vandekerckhove, J., Forstmann, B. U., Keuleers, E., Brysbaert, M., \& Wagenmakers, E.-J. (2012). Testing theories of post-error slowing. Attention, Perception \& Psychophysics, 74(2), 454-465. https://doi.org/10.3758/s13414-011-0243-2

Fievez, F., Derosiere, G., Verbruggen, F., \& Duque, J. (2021). Post-error slowing reflects the joint impact of adaptive and maladaptive processes during decision making [Preprint]. Neuroscience. https://doi.org/10.1101/2021.12.22.473805 
Franklin, D. W., \& Wolpert, D. M. (2011). Computational mechanisms of sensorimotor control. Neuron, 72(3), 425-442. https://doi.org/10.1016/j.neuron.2011.10.006

Gigerenzer, G., \& Gaissmaier, W. (2011). Heuristic Decision Making. Annual Review of Psychology, 62(1), 451-482. https://doi.org/10.1146/annurev-psych-120709-145346

Hagura, N., Haggard, P., \& Diedrichsen, J. (2017). Perceptual decisions are biased by the cost to act. ELife, 6, e18422. https://doi.org/10.7554/eLife.18422

Haith, A. M., Reppert, T. R., \& Shadmehr, R. (2012). Evidence for Hyperbolic Temporal Discounting of Reward in Control of Movements. Journal of Neuroscience, 32(34), 11727-11736. https://doi.org/10.1523/JNEUROSCI.0424-12.2012

Jentzsch, I., \& Dudschig, C. (2009). Short Article: Why do we slow down after an error? Mechanisms underlying the effects of posterror slowing. Quarterly Journal of Experimental Psychology, 62(2), 209-218. https://doi.org/10.1080/17470210802240655

King, J. A., Korb, F. M., von Cramon, D. Y., \& Ullsperger, M. (2010). Post-error behavioral adjustments are facilitated by activation and suppression of task-relevant and taskirrelevant information processing. The Journal of Neuroscience: The Official Journal of the Society for Neuroscience, 30(38), 12759-12769. https://doi.org/10.1523/JNEUROSCI.3274-10.2010

Laming, D. (1979). Choice reaction performance following an error. Acta Psychologica, 43(3), 199-224. https://doi.org/10.1016/0001-6918(79)90026-X

Marcos, E., Cos, I., Girard, B., \& Verschure, P. F. M. J. (2015). Motor Cost Influences Perceptual Decisions. PLOS ONE, 10(12), e0144841. https://doi.org/10.1371/journal.pone.0144841 
561

562

563

564

565

566

567

568

569

570

571

572

573

574

575

576

577

578

579

580

581

582

Morel, P., Ulbrich, P., \& Gail, A. (2017). What makes a reach movement effortful? Physical effort discounting supports common minimization principles in decision making and motor control. PLOS Biology, 15(6), e2001323. https://doi.org/10.1371/journal.pbio.2001323

Notebaert, W., Houtman, F., Opstal, F. V., Gevers, W., Fias, W., \& Verguts, T. (2009). Posterror slowing: An orienting account. Cognition, 111(2), 275-279. https://doi.org/10.1016/j.cognition.2009.02.002

Purcell, B. A., \& Kiani, R. (2016). Neural Mechanisms of Post-error Adjustments of Decision Policy in Parietal Cortex. Neuron, 89(3), 658-671. https://doi.org/10.1016/j.neuron.2015.12.027

Rabbitt, P., \& Rodgers, B. (1977). What does a man do after he makes an error? An analysis of response programming. Quarterly Journal of Experimental Psychology, 29(4), 727-743. https://doi.org/10.1080/14640747708400645

Ratcliff, R., Smith, P. L., Brown, S. D., \& McKoon, G. (2016). Diffusion Decision Model: Current Issues and History. Trends in Cognitive Sciences, 20(4), 260-281. https://doi.org/10.1016/j.tics.2016.01.007

Reynaud, A. J., Saleri Lunazzi, C., \& Thura, D. (2020). Humans sacrifice decision-making for action execution when a demanding control of movement is required. Journal of Neurophysiology, 124(2), 497-509. https://doi.org/10.1152/jn.00220.2020

Saleri Lunazzi, C., Reynaud, A. J., \& Thura, D. (2021). Dissociating the Impact of Movement Time and Energy Costs on Decision-Making and Action Initiation in Humans. Frontiers in Human Neuroscience, 15, 715212. https://doi.org/10.3389/fnhum.2021.715212 
Shadmehr, R., \& Ahmed, A. A. (2020). Vigor: Neuroeconomics of Movement Control. The MIT Press. https://doi.org/10.7551/mitpress/12940.001.0001

Shadmehr, R., Orban de Xivry, J. J., Xu-Wilson, M., \& Shih, T.-Y. (2010). Temporal Discounting of Reward and the Cost of Time in Motor Control. Journal of Neuroscience, 30(31), 10507-10516. https://doi.org/10.1523/JNEUROSCI.1343-10.2010

Shadmehr, R., Reppert, T. R., Summerside, E. M., Yoon, T., \& Ahmed, A. A. (2019). Movement Vigor as a Reflection of Subjective Economic Utility. Trends in Neurosciences, 42(5), 323-336. https://doi.org/10.1016/j.tins.2019.02.003

Thura, D. (2020). Decision urgency invigorates movement in humans. Behavioural Brain Research, 382, 112477. https://doi.org/10.1016/j.bbr.2020.112477

Thura, D. (2021). Reducing behavioral dimensions to study brain-environment interactions. Behavioral and Brain Sciences, 44. https://doi.org/10.1017/S0140525X21000169

Thura, D., Cos, I., Trung, J., \& Cisek, P. (2014). Context-dependent urgency influences speedaccuracy trade-offs in decision-making and movement execution. The Journal of Neuroscience: The Official Journal of the Society for Neuroscience, 34(49), 1644216454. https://doi.org/10.1523/JNEUROSCI.0162-14.2014

Thura, D., Guberman, G., \& Cisek, P. (2017). Trial-to-trial adjustments of speed-accuracy tradeoffs in premotor and primary motor cortex. Journal of Neurophysiology, 117(2), 665683. https://doi.org/10.1152/jn.00726.2016

Urai, A. E., de Gee, J. W., Tsetsos, K., \& Donner, T. H. (2019). Choice history biases subsequent evidence accumulation. ELife, 8, e46331. https://doi.org/10.7554/eLife.46331 
604 Yoon, T., Geary, R. B., Ahmed, A. A., \& Shadmehr, R. (2018). Control of movement vigor and 605 decision making during foraging. Proceedings of the National Academy of Sciences, 